Nicht kleinzelliges Lungenkarzinom

\section{Auch ältere Patienten profitieren von Multi-Target-Inhibitor}

Beim metastasierten Lungenkarzinom kommt es bei der Therapie nicht nur auf die Lebensverlängerung, sondern auch auf eine gute Lebensqualität an. Entscheidend für die Wahl der Therapie sind die Begleiterkrankungen, der Allgemeinzustand und der Patientenwille. "Wir müssen uns gut anschauen, welche Patienten wir auswählen“, erklärte Corinna Eschbach, Hamburg. „Aber das Alter allein ist kein Kriterium für eine bestimmte Therapie."

Dass eine platinbasierte Kombinationstherapie auch bei älteren Patienten mit einem fortgeschrittenen nicht kleinzelligen Lungenkarzinom durchaus sinnvoll ist, dazu liegen gute Daten vor. Eine Subgruppenanalyse der Studie ECOG 1594 zeigt, dass ältere Patienten ebenso von dieser Kombination profitieren wie jüngere. Die Lebensverlängerung war in beiden Altersgruppen vergleichbar, obwohl bei älteren Patienten mehr Toxizitäten auftraten.

Einen hohen Stellenwert für die Therapiewahl hat darüber hinaus die Histologie des Tumors. Betrachtet man die Gesamtgruppe der nicht kleinzelligen Lungenkarzinome hat die Kombination Pemetrexed (Alimta ${ }^{\circ}$ ) plus Cisplatin eine ähnlich gute Wirksamkeit wie die Standardtherapie mit Gemcitabin plus Cisplatin. Unterscheidet man zwischen den verschiedenen histologischen Typen, dann stellt sich heraus, dass Patienten mit einem Adenokar- zinom oder einem großzelligen Lungenkarzinom länger leben, wenn sie eine Kombinationstherapie mit Pemetrexed erhalten. Den Ergebnissen einer Phase-IIIStudie zufolge stieg das mediane Überleben bei diesen Patienten von 10,9 Monaten unter Gemcitabin/Cisplatin auf 12,6 Monate unter der Pemetrexed-Kombination. Und auch bei großzelligen
Lungenkarzinomen ist der MultiTarget-Inhibitor überlegen, hier betrug der Überlebensvorteil 10,4 Monate vs. 6,7 Monate.

Dass diese Kombination auch gut bei älteren Patienten zum Einsatz kommen kann, bestätigten Subgruppenanalysen: Die Therapie war bei Patienten über 65 Jahren vergleichbar effektiv wie bei jüngeren Patienten. Auch in dieser Altersgruppe nutzte Pemetrexed vor allem Patienten mit Nichtplattenepithelkarzinomen. Das Überleben stieg bei den über 65-jährigen Patienten mit Nichtplattenepithelkarzinomen von 9,8 Monaten unter Gemcitabin/ Cisplatin auf 11,3 Monate unter Pemetrexed/Cisplatin. jn

Symposium im Rahmen des Kongresses der Deutschen Gesellschaft für Pneumologie am 8. April 2011 in Dresden; Veranstalter: Lilly Oncology

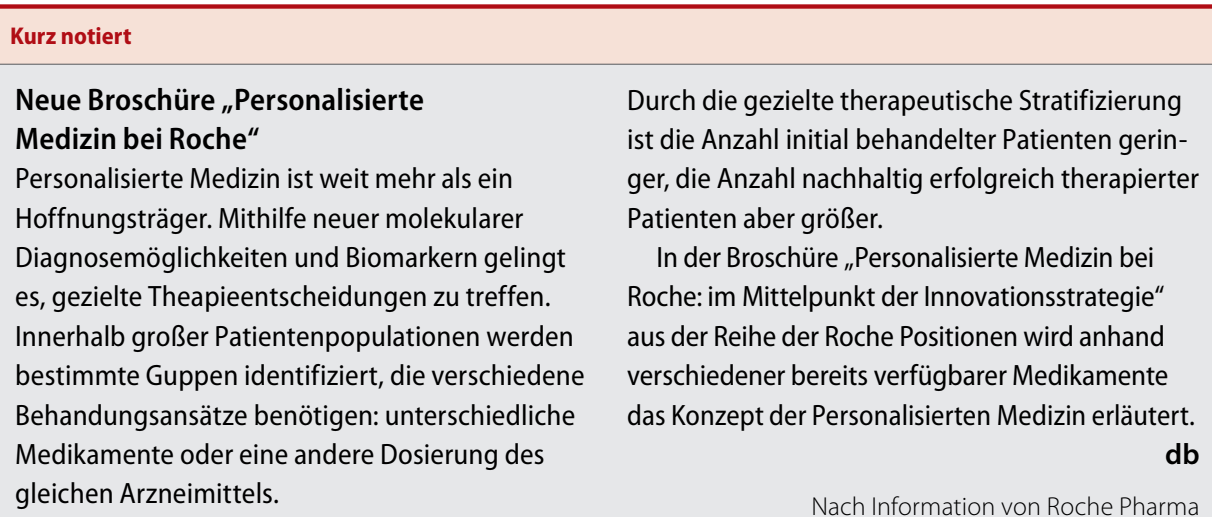

